\title{
Atmospheric excitation of the Earth's free core nutation (Research Note)
}

\author{
S. B. Lambert
}

Royal Observatory of Belgium, 1180 Brussels, Belgium

e-mail: s.lambert@oma.be

Received 13 June 2006 / Accepted 13 July 2006

ABSTRACT

\begin{abstract}
Aims. This study investigates the time-varying excitation of the Earth's retrograde free core nutation (RFCN) by atmospheric pressure variations.

Methods. The amplitude of the RFCN resulting from diurnal pressure variations in the external fluid layers is derived. The case of atmospheric excitation is investigated using the National Center for Environmental Prospect/National Center for Atmospheric Research (NCEP/NCAR) Reanalysis project data set.

Results. The diurnal atmospheric excitation is about half that required to explain the observed RFCN amplitude. Moreover, the time variability of the atmospheric noise does not account for the observed variability of the RFCN amplitude over the time period 1984-2006. Possible sources of error like the pressure coefficient, the RFCN quality factor, or the time varying RFCN apparent period are investigated but cannot account for the discrepancy.

Conclusions. Improvements are needed in atmospheric modeling at diurnal frequencies. Until this is achieved, the physical link between the observed RFCN and its atmospheric excitation, especially concerning its time variability, will remain unclear.
\end{abstract}

Key words. reference systems - Earth

\section{Introduction}

Retrograde free core nutation $(\mathrm{RFCN})$ is a free rotational mode of the Earth, associated with the ellipsoidal liquid core rotating inside the visco-elastic mantle. The signature of this free mode on the Earth's figure axis observed from a space-fixed reference frame is a retrograde motion (opposite to the Earth's rotation) that reaches an amplitude of about $200 \mu$ as, variable in time, and with a period estimated at -430.23 sidereal days (Herring et al. 2002) with a variable phase (see for instance Roosbeek et al. 1999; Vondrák et al. 2005).

The excitation mechanism of the RFCN is still an open question (Dehant et al. 2003). Several studies have shown evidence of a source of excitation in the surface geophysical fluids (atmosphere, oceans). The RFCN is comparable to the Chandler wobble, the other free rotational mode of the Earth, occurring with a period of 433 days in the Earth-fixed reference frame. Gross (2000) showed that atmospheric and ocean bottom pressure variations could excite the Chandler wobble. Similarly, the long-term modulation of the diurnal signal in geophysical fluids could drive the amplitude variability and the phase changes of the free core nutation.

Understanding the excitation mechanism of the RFCN is a challenging question for the geosciences community. Direct implications are found from the determination of Earth's interior parameters, in atmospheric and oceanic sciences, or operational applications like the prediction of Earth orientation parameters computed at the International Earth Rotation and Reference Systems Service (IERS), a crucial activity for high precision positioning or space navigation.

Meteorological data (atmospheric pressure and winds) are provided on a regular basis by several agencies. The 6-h time resolution of some of these data sets allows one to explore the diurnal and sub-diurnal signal likely able to contribute to the RFCN excitation. Additionally, the RFCN is directly observable through very long baseline radio interferometry (VLBI) measurements. Figure 1 displays the RFCN oscillation projected on the $X$ and $Y$ axes of the conventional celestial reference frame and in a 3-dimensional representation (the signal is extracted by a least-squares fit from the IERS EOP C 04 data set, a combination of several VLBI data sets made available at the IERS Earth Orientation Center).

In this study, we establish a link between excitation in the fluid layers and the resulting amplitude of the RFCN, via the equations of Brzeziński (1994) with the assumption that this excitation can be modeled by white noise (Sect. 2). In Sect. 3, the case of atmospheric excitation is studied and discussed: the RFCN amplitude predicted from the meteorological data set is compared to VLBI results and possible sources of error are pointed out.

\section{Theory}

Brzeziński (1994) derived the time-domain relation between the external fluid layer excitation $\chi^{\prime}$ (e.g., coming from the atmosphere, oceans, continental water), expressed in the spacefixed reference frame, and the induced nutational motion $\eta=$ $\Delta \psi \sin \epsilon_{0}+\mathrm{i} \Delta \epsilon$ of the Earth's figure axis:

$$
\begin{aligned}
\frac{\mathrm{d}^{2} \eta}{\mathrm{d} t^{2}}-\mathrm{i}\left(\sigma_{\mathrm{c}}^{\prime}+\sigma_{\mathrm{f}}^{\prime}\right) \frac{\mathrm{d} \eta}{\mathrm{d} t}-\sigma_{\mathrm{c}}^{\prime} \sigma_{\mathrm{f}}^{\prime} \eta= & -\mathrm{i} \sigma_{\mathrm{c}}(1+a) \frac{\mathrm{d} \chi^{\prime}}{\mathrm{d} t} \\
& -\sigma_{\mathrm{c}}\left(\sigma_{\mathrm{c}}^{\prime}+a \sigma_{\mathrm{f}}^{\prime}\right) \chi^{\prime}
\end{aligned}
$$

where $\sigma_{\mathrm{f}}^{\prime}=\sigma_{0}^{\prime}\left(1-\mathrm{i} / 2 Q_{\mathrm{f}}^{\prime}\right)$ is the complex RFCN frequency $\left(\sigma_{0}^{\prime}\right.$ is negative), $\sigma_{\mathrm{c}}$ is the complex Chandler frequency, and $a$ is a 

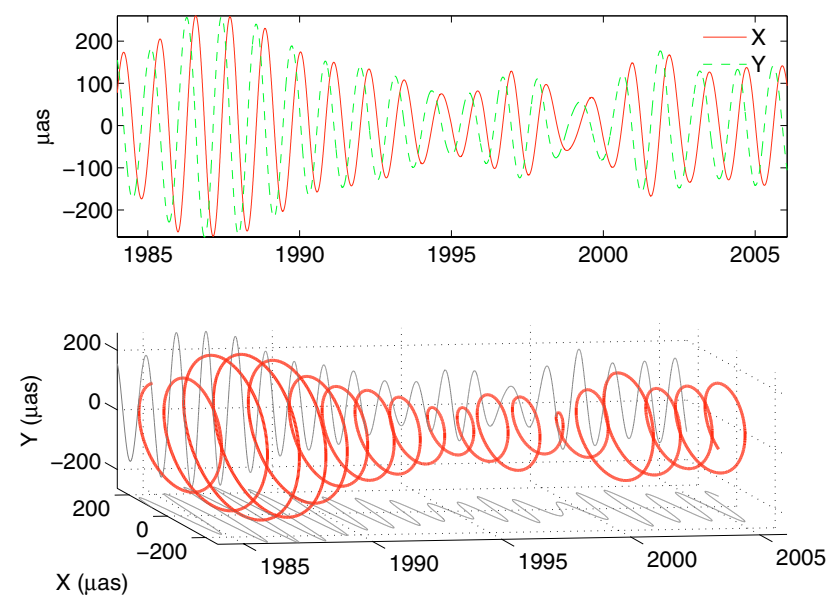

Fig. 1. Least-squares fit and 3D representation of the free core nutation based on the combined IERS EOP C 04 data.

dimensionless coefficient expressing the response of the nutation to the pressure and wind excitations $\left(a_{\mathrm{p}}=9.509 \times 10^{-2}\right.$ and $\left.a_{\mathrm{w}}=5.489 \times 10^{-4}\right)$. In the following, the primed quantities are expressed in the space-fixed reference frame, as opposed to the Earth-fixed one. The celestial excitation $\chi^{\prime}$ is a dimensionless quantity proportional to the angular momentum (see e.g., Barnes et al. 1983) and deduced from its terrestrial counterpart $\chi$ by the rotation $\chi^{\prime}=-\chi \exp (i \Phi)$ where $\Phi$ is the Greenwich sidereal time (Bizouard et al. 1998).

Turning (2) into the frequency domain, one gets

$\eta\left(\sigma^{\prime}\right)=T\left(\sigma^{\prime}\right) \chi^{\prime}\left(\sigma^{\prime}\right)$

wherein the transfer function $T$ is given by

$T\left(\sigma^{\prime}\right)=\frac{\sigma_{\mathrm{c}}}{\sigma_{\mathrm{c}}^{\prime}-\sigma^{\prime}}+\frac{a \sigma_{\mathrm{c}}}{\sigma_{\mathrm{f}}^{\prime}-\sigma^{\prime}}$.

The frequency $\sigma^{\prime}$ is typically $1 / 300$ cycle per day (cpd), while the Chandler frequency in space is close to $1 \mathrm{cpd}$. The first term of the transfer function is therefore close to 1 . The RFCN frequency is roughly $-1 / 430 \mathrm{cpd}$, so that the difference $\sigma_{\mathrm{f}}^{\prime}-\sigma^{\prime}$ appears to be around $1 / 1000 \mathrm{cpd}$. For a pressure excitation, the second term of the transfer function is close to 100 . The first term accounts therefore for $1 \%$ of the total effect and is negligible considering that the effects on the nutation we are looking for reach no more than a few hundred micro arc seconds. In the case of a wind excitation, the second term is smaller than the same term for a pressure excitation by a factor of 100 and is therefore of the same order of magnitude as the first term. However, the wind contribution remains marginal and will not be considered in the following. In this assumption (i) the above equations are not sensitive to the Chandler dissipation and one can take a real Chandler frequency of $\sigma_{\mathrm{c}}=1 / 433.3 \mathrm{cpd}$; and (ii) for $\sigma^{\prime}$ close to $\sigma_{0}^{\prime}$, only the second term of the transfer function is relevant and will be considered hereafter.

One can write the RFCN amplitude as a damped exponential function:

$\eta=A \mathrm{e}^{\mathrm{i} \sigma_{0}^{\prime} t} \mathrm{e}^{-\alpha t}$,

where $\alpha=\left|\sigma_{0}^{\prime}\right| / 2 Q_{\mathrm{f}}^{\prime}$ expresses the damping and $A$ is a complex amplitude. Note that the damping factor $Q_{\mathrm{f}}^{\prime}$ is considered in the space-fixed reference frame and is deduced from its counterpart $Q_{\mathrm{f}}=-Q_{\mathrm{f}}^{\prime} \sigma_{0} / \sigma_{0}^{\prime}$ in the Earth-fixed reference frame.
For a white noise process occurring in the fluid layers, the spectral density $\chi^{\prime}\left(\sigma^{\prime}\right)$ is equal to a constant value $\chi_{0}^{\prime}$ over a frequency band $\Delta$ around $\sigma_{0}^{\prime}$. The corresponding power transferred to the nutation is:

$$
\begin{aligned}
P_{\Delta} & =\int_{\sigma_{0}^{\prime}-\Delta / 2}^{\sigma_{0}^{\prime}+\Delta / 2} \eta\left(\sigma^{\prime}\right) \eta^{*}\left(\sigma^{\prime}\right) \mathrm{d} \sigma^{\prime} \\
& =\chi_{0}^{\prime 2} \int_{\sigma_{0}^{\prime}-\Delta / 2}^{\sigma_{0}^{\prime}+\Delta / 2} T\left(\sigma^{\prime}\right) T^{*}\left(\sigma^{\prime}\right) \mathrm{d} \sigma^{\prime} .
\end{aligned}
$$

After some algebra and dropping the first term in (3), one gets from (6)

$P_{\Delta}^{\left(\chi^{\prime}\right)}=\frac{2 \chi_{0}^{\prime 2} a_{\mathrm{p}}^{2} \sigma_{\mathrm{c}}^{2}}{\alpha} \operatorname{atan}\left(\frac{\Delta}{2 \alpha}\right)$.

The contribution of a fluid layer excitation to the RFCN amplitude can be retrieved considering the assumed time-domain expression (4) of the RFCN. By Fourier-transforming (4), one gets the spectral density

$\eta\left(\sigma^{\prime}\right)=\frac{2 \sqrt{2 \pi} A}{\alpha+\mathrm{i}\left(\sigma^{\prime}-\sigma_{0}^{\prime}\right)}$

leading, using (5), to the power

$P_{\Delta}^{(\eta)}=\frac{16 \pi A^{2}}{\alpha} \operatorname{atan}\left(\frac{\Delta}{2 \alpha}\right)$

that can be equated to the corresponding power (7) coming from the fluid layer, thus obtaining the reconstructed amplitude $A$ of RFCN coming from the whole fluid layer spectrum:

$A=\frac{\chi_{0}^{\prime} a_{\mathrm{p}} \sigma_{\mathrm{c}}}{2 \pi \sqrt{2}}$

Note that $A$ does not depend on $\Delta$, meaning that prograde frequencies are able to influence the RFCN, although the RFCN is a retrograde process.

The remaining problem is to get an estimate of the spectral density $\chi_{0}^{\prime}$ of the white noise present in the fluid layers. The specific case of the atmosphere will be explored in the next section.

\section{The excitation from the atmosphere}

We use atmospheric angular momentum time series made available at the IERS Special Bureau for the Atmosphere (chaired by D. Salstein, at Atmospheric and Environmental Research, Inc., Mass., see Salstein et al. 1997) spanning 1970-2006, computed within the framework of the National Center for Environmental Prospect/National Center for Atmospheric Research (NCEP/NCAR) Reanalysis project (Kalnay et al. 1993). Note that the comparison to reliable VLBI data will only be possible for 1984-2006. The atmospheric data are converted to the celestial frame and then resampled at 10-day intervals using a Gaussian interpolation, thereby removing the diurnal signal, which is irrelevant since we consider only the excitation on annual time scales. The data are plotted in Fig. 2 along with a complex spectrum and a wavelet spectrum.

The complex spectrum successively shows the prograde waves $S_{1}$ (1 cycle per year (cpy)), $S_{2}$ (2 cpy) and $S_{3}$ (3 cpy). A retrograde annual peak appears but is poorly significant considering the noise level. From the wavelet spectrum, one sees that the prograde annual term admits a variable amplitude, yielding several maxima around 1977, 1990, 1995 and 1999, and 

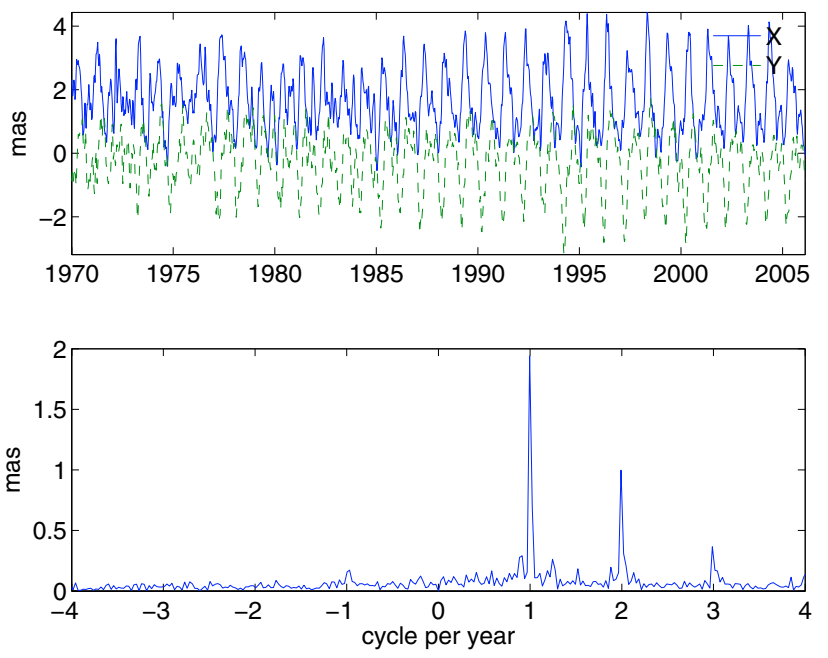

mas
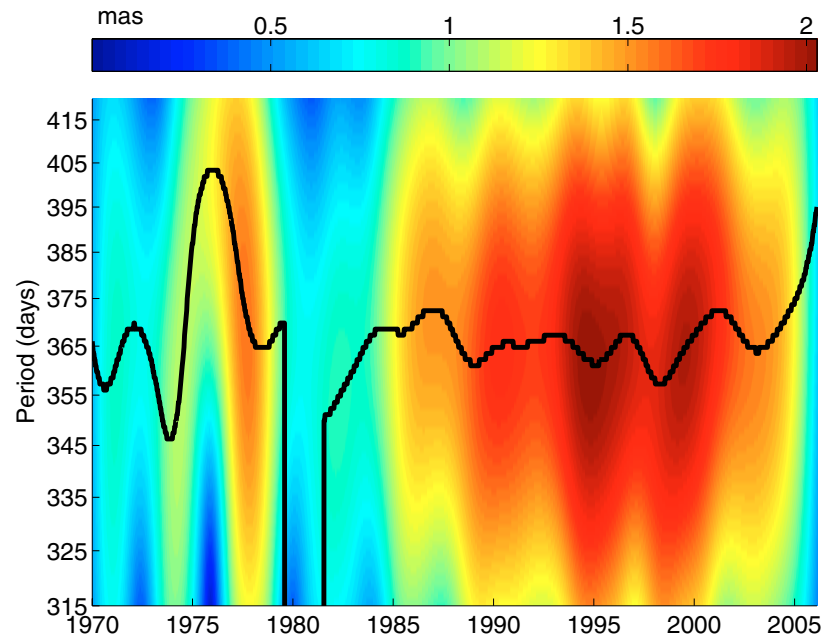

Fig. 2. Celestial atmospheric excitation function (top), its complex spectrum (middle) and the annual prograde part of the wavelet spectrum (bottom, the black, thick line represents the ridge, i.e., the period for which the instantaneous amplitude is maximal).

a variable phase, making the apparent period vary between 345 and 405 days, the latter period being reached around 1977. Note that the location of the ridge does not give a reliable estimate of the period for too weak amplitudes (this is for instance the case around 1980).

The atmospheric excitation, from which the mean annual, semi-annual and tri-annual variations are removed, is then modeled by an autoregressive process which optimal order is determined by minimizing the Akaike final prediction error criterion. The order 0 coefficient gives the weight of the white noise in the total variance of the signal. The reconstructed amplitude $A$ of the RFCN is deduced from (10). Local estimates of $\chi_{0}^{\prime}$ and $A$ are thus realized on successive subsets of 100 days by applying a sliding window to the original data set. Results are displayed in Fig. 3. Note that taking another size for the window would not significantly change the results.

Comparing the observed RFCN amplitude (Fig. 1) and the reconstructed amplitude (Fig. 3) from 1984-2006, it appears that the atmospheric contribution is too small by a factor of one-half to explain the observed amplitude. This implies (i) a lack of power in the atmospheric data; or (ii) some concurrent effects within or outside the geophysical fluid layers.

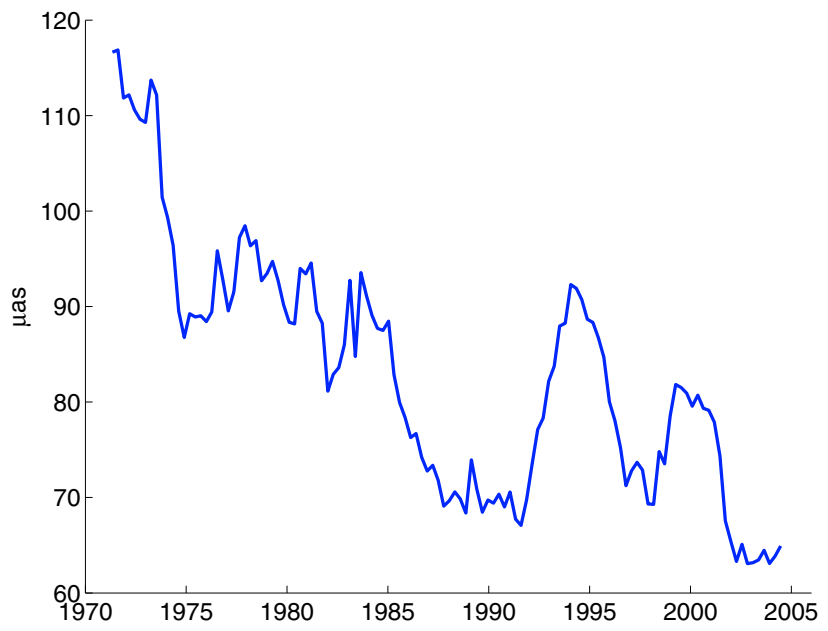

Fig. 3. The reconstructed RFCN amplitude $A$ computed from the atmospheric excitation.

Comparing now the wavelet spectrum in Figs. 2 with 3, one can see that maxima in the amplitude of $S_{1}$ correspond to pulses in the reconstructed amplitude $A$, especially for the pulses occurring in 1995 and 1999. The amplitude of these pulses is between 10 and $30 \mu$ as, therefore too weak to account for the observed time variability of the RFCN amplitude, which is about $100 \mu$ as (see Fig. 1).

Several possible sources of errors should be addressed. The first one is the value of the coefficient $a_{\mathrm{p}}$ expressing the response of the nutation to the pressure loading. The relative uncertainty of this coefficient is around 3\%, as mentioned by Brzeziński (1994), and does not constitute a large source of error in the amplitude $A$.

The RFCN quality factor $Q_{\mathrm{f}}$ estimated from various data sets and techniques (e.g., VLBI, superconducting gravimeters) can vary widely, from a thousand to several hundreds of thousands, with comparable uncertainties (see for instance Hinderer et al. 2000, for a review). However, the amplitude $A$ induced by the fluid layers does not depend on this quality factor, thus removing sources of error coming from the choice of $Q_{\mathrm{f}}$.

There remains the uncertainty from atmospheric variations on diurnal time scales. Yseboodt et al. (2002) compared the time variability of the main diurnal frequencies extracted from various atmospheric angular momentum data sets and found differences of $100 \%$ from one model to another. Similar issues were brought up by de Viron et al. (2005), who compared the LMD model (purely dynamical, no data assimilation) with the NCEP and ECMWF on a short time span (less than 2 years) and showed that the three models differ strongly on diurnal time scales: although the amplitudes of the prograde $S_{1}$ are in agreement, the phases show differences of several tens of degrees. They showed also that $S_{1}$ is the wave for which the agreement between models is the best, implying that the quality of the modeling for the rest of the spectrum is poorer. In our case, since one is looking for a signal centered on the RFCN frequency, for which, according to the Fourier spectrum in Fig. 2, the noise level is less than $10 \%$ of the $S_{1}$ amplitude, one cannot expect that the time variation of the noise magnitude rendered by any spectral analysis would be statistically significant.

Variations of the apparent period of the annual prograde term can also be responsible for pulses in the excitation of the RFCN. Indeed, if the period of the excitation is far from the RFCN frequency, the transfer function enhances the amplitude differently. 
A minimum of period of the annual prograde term thus leads to a lower excitation of the RFCN, and inversely. Around 1977, the apparent period detected on the wavelet spectrum increases significantly to 405 days. The relative effect on $A$ can be evaluated from the transfer function and is close to $5 \%$, corresponding to a variation of about $5 \mu$ as for an amplitude of $100 \mu$ as. This could partly explain some of the variations of $A$ observed in Fig. 3 around 1977. However, changes in the period during the 1990's are unable to account for variations in $A$.

\section{Conclusion}

This work provides qualitative insights into the excitation of the Earth's retrograde free core nutation by atmospheric pressure variations. We use a formalism based on Brzeziński's (1994) equations, linking the amplitude of the space motion of the Earth's figure axis to the atmospheric excitation based on the assumption that the atmospheric excitation can be modeled by white noise.

Globally, the atmospheric contribution to the RFCN accounts for half of the observed RFCN amplitude. However, the time variability of the RFCN signal, as observed by VLBI, is not explained by the time-varying noise in the atmosphere. The variations of the latter are too weak and not statistically significant considering the uncertainty attached to the atmospheric data in the diurnal band.

Improvements are needed in atmospheric modeling to improve the reliability of the atmospheric data at diurnal frequencies. Until this is achieved, the physical link between the observed RFCN and its atmospheric excitation, especially concerning its time variability, will remain unclear. Further research is also necessary to extend the study to other geophysical fluid layers, especially to ocean dynamics. A lack of oceanic data in the diurnal band does not allow us to investigate their effects on the RFCN, although they are expected to be significant.

Acknowledgements. The author is grateful to Prof. V. Dehant, Drs. O. de Viron, C. Bizouard, and C. Le Poncin-Lafitte for valuable discussions, and to the anonymous referee for useful suggestions in improving the manuscript.

\section{References}

Barnes, R. T. H., Hide, R., White, A. A., \& Wilson, C. A. 1983, Proc. R. Soc. Lond., A 387, 31

Bizouard, C., Brzeziński, A., \& Petrov, S. D. 1998, J. Geod., 72, 561

Brzeziński, A. 1994, Manuscripta Geodaetica, 19, 157

Dehant, V., Feissel-Vernier, M., de Viron, et al. 2003, J. Geophys. Res., 108(B5)

Gross, R. S. 2000, Geophys. Res. Lett., 27(15), 2329

Herring, T. A., Mathews, P. M., \& Buffett, B. A. 2002, J. Geophys. Res., 107(B4)

Hinderer, J., Boy, J.-P., Gegout, P., et al. 2000, PEPI, 117(4), 37

Kalnay, E., Kanamitsu, M., Kistler, R., et al. 1996, Bull. Amer. Meteor. Soc., 76, 437471

Roosbeek, F., Defraigne, P., Feissel, M., \& Dehant, V. 1999, Geophys. Res. Lett., 26(1), 131

Salstein, D. A., Kann, D. M., Miller, A. J., \& Rosen, R. D. 1993, Bull. Amer. Meteorol. Soc., 74, 64

de Viron, O., Schwarzbaum, G., Lott, F., \& Dehant, V. 2005, J. Geophys. Res., 110(B11)

Vondrák, J., Weber, R., \& Ron, C. 2005, A\&A, 444, 297

Yseboodt, M., de Viron, O., Chin, T. M., \& Dehant, V. 2002, J. Geophys. Res., 107(B2) 